\title{
Testsofa Design Details and Theory of Operation
}

Carl H Miller

March 2016

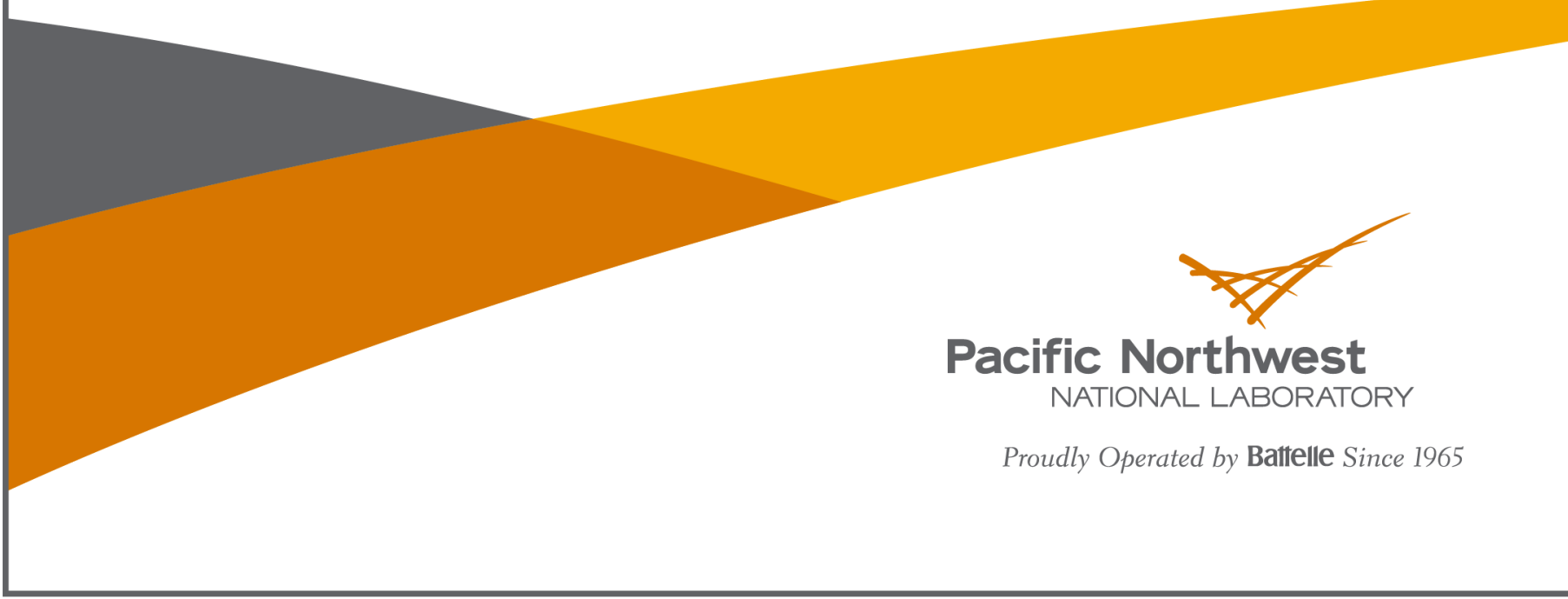




\title{
DISCLAIMER
}

This report was prepared as an account of work sponsored by an agency of the United States Government. Neither the United States Government nor any agency thereof, nor Battelle Memorial Institute, nor any of their employees, makes any warranty, express or implied, or assumes any legal liability or responsibility for the accuracy, completeness, or usefulness of any information, apparatus, product, or process disclosed, or represents that its use would not infringe privately owned rights. Reference herein to any specific commercial product, process, or service by trade name, trademark, manufacturer, or otherwise does not necessarily constitute or imply its endorsement, recommendation, or favoring by the United States Government or any agency thereof, or Battelle Memorial Institute. The views and opinions of authors expressed herein do not necessarily state or reflect those of the United States Government or any agency thereof.

\author{
PACIFIC NORTHWEST NATIONAL LABORATORY \\ operated by \\ BATTELLE \\ for the \\ UNITED STATES DEPARTMENT OF ENERGY \\ under Contract DE-AC05-76RL01830
}

Printed in the United States of America

Available to DOE and DOE contractors from the

Office of Scientific and Technical Information,

P.O. Box 62, Oak Ridge, TN 37831-0062;

ph: (865) 576-8401

fax: $(865)$ 576-5728

email: reports@adonis.osti.gov

\footnotetext{
Available to the public from the National Technical Information Service, U.S. Department of Commerce, 5285 Port Royal Rd., Springfield, VA 22161 ph: (800) 553-6847 fax: $(703) 605-6900$

email: orders@ntis.fedworld.gov

online ordering: http://www.ntis.gov/ordering.htm
}

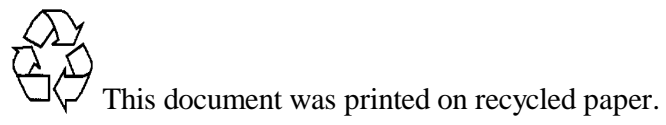




\section{Testsofa Design Details and Theory of Operation}

Carl H Miller

March 2016

Prepared for

the U.S. Department of Energy

under Contract DE-AC05-76RL01830

Pacific Northwest National Laboratory

Richland, Washington 99352 


\section{Testsofa Design Details and Theory of Operation}

\section{Overview}

The Testsofa is used to explore the functioning of an integrated circuit. It characterizes pin functions via pin profiling, then serves as an interface to a Boss server which creates a finite state machine (FSM) model using branch exploration. This exploration is done via a stimulus/response process, which will be explained in the Internal Logic Testing section.

\section{Pin Profiling}

The pin profiling process is done in four phases:

Phase 1: For the scan process, the CPU sequentially measures voltages for each of the UUT pins. For each pin, the pin is first grounded via the latches and the grounding transistor. The rest of the pins are pulled toward the power voltage through their pull-up resistors. The voltages on the non-grounded pins are measured by the ADCs via the SPI port, and the sum of the voltages is stored in the array pinVoltSumPower. Next, the same pin is left pulled-high while the other pins are grounded, and the voltage on that pin is stored in the array pinVoltSumGround. The CPU repeats this process for all UUT pins.

Phase 2: The analysis process of the pin-profile algorithm starts by determining the power and ground pins. The power pin will be the pin corresponding to the lowest value in the pinVoltSumPower array and the ground pin will the pin corresponding to the lowest value in the pinVoltSumGround array. In both cases the lowest voltage results from the highest current drawn through the pull-up resistor(s).

Phase 3: With the power and ground pins determined, the algorithm then calculates the average voltage of the remaining pins. If the pulled-up ground pin voltage is less than the average voltage, the input pins will have pinVoltSumPower values less than the average and the output pins will have pinVoltSumPower values greater than the average. If the pulled-up ground pin voltage is greater than the average, the input and output pin voltages will have the opposite relationships to the average voltage.

Phase 4: With the input and output pins determined, the clock and reset pins are found by setting the input pins with all possible combinations for the number of inputs. For each combination, each of the input pins is toggled between 0 and 1 . The output is assumed to start at zero, the combination and pin toggled which result in a non-zero output are the clocking state and clock pin, respectively. Next, the reset pin is searched within the clocking state. Each input pin is toggled, and when the outputs go back to zero, the pin toggled will be the reset pin and its toggled state will be the active-level for the reset.

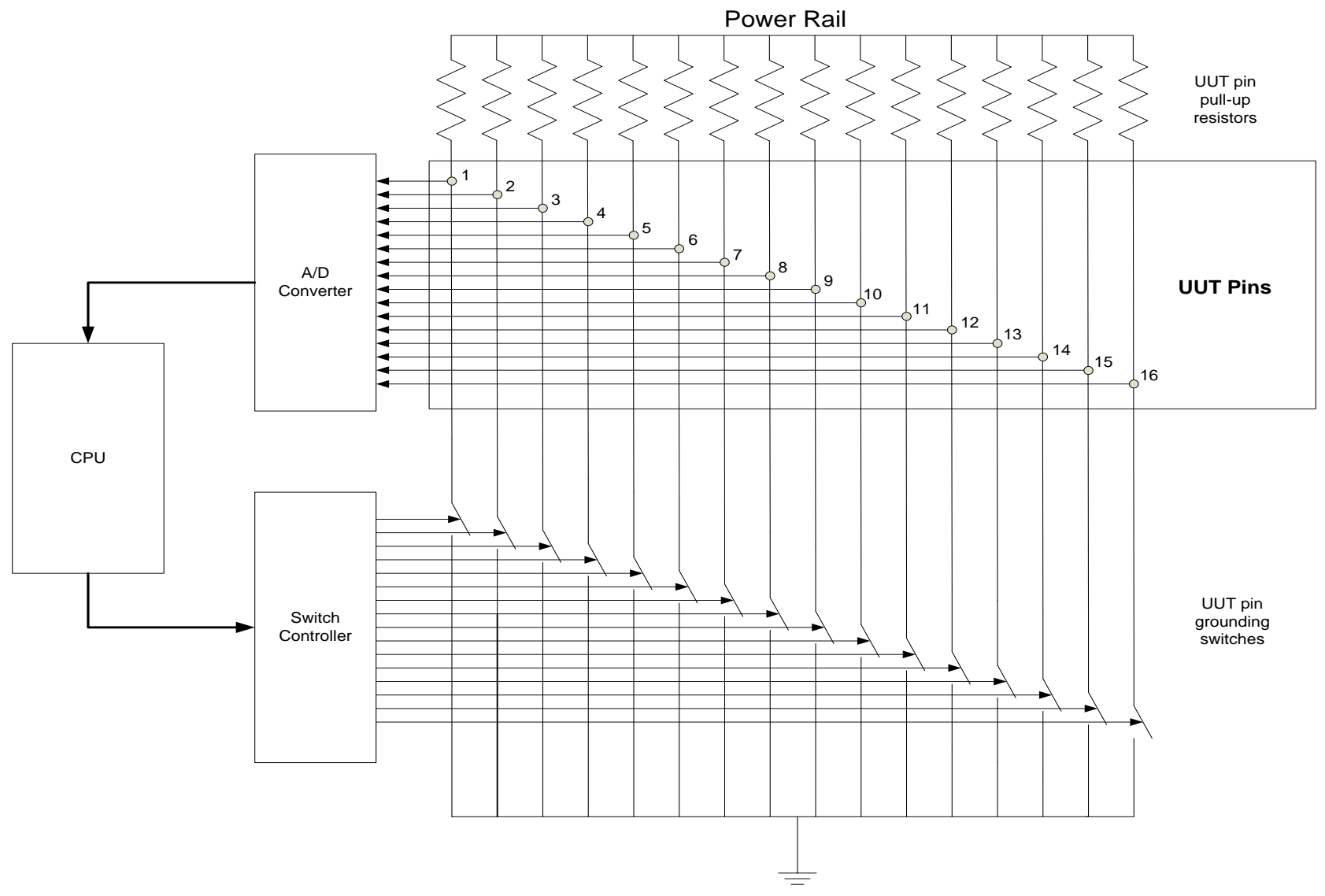




\section{Internal Logic Testing:}

Commands and requests are sent from the Boss (see next section) to the FPGA-based Tester via the CPU. The Tester's CPU Interface/Process Controller receives these, directs the testing accordingly, and sends statuses and the response data back to the CPU. The statuses are used to indicate quantities of data available. The Tester also contains a UUT Interface and separate input and output 2 Kbyte FIFOs to connect the Process Controller to the UUT Interface while allowing some operational independence between them.

When the stimulus request is first received or created by the CPU, the CPU signals the Tester to clear its FIFOs and waits until they are cleared. Next, using the pin-profile, the CPU creates the Stimulus/Response Mask and connects the power and ground pins. The stimulus mask and write command/block size/data are then sent to the Tester, which places the stimulus data into the input FIFO. Whenever the Process Controller detects that the input FIFO contains data, it enables the UUT Interface, which pulls stimulus data from the input FIFO, applies it to the UUT, and pushes the response data into the output FIFO. The CPU polls the Tester for the status of the data remaining in the input FIFO until the FIFO is empty, and then sends the read command/block size to the Tester. Finally, the CPU reads the response data from the Tester's output FIFO, and converts the response data into state data.

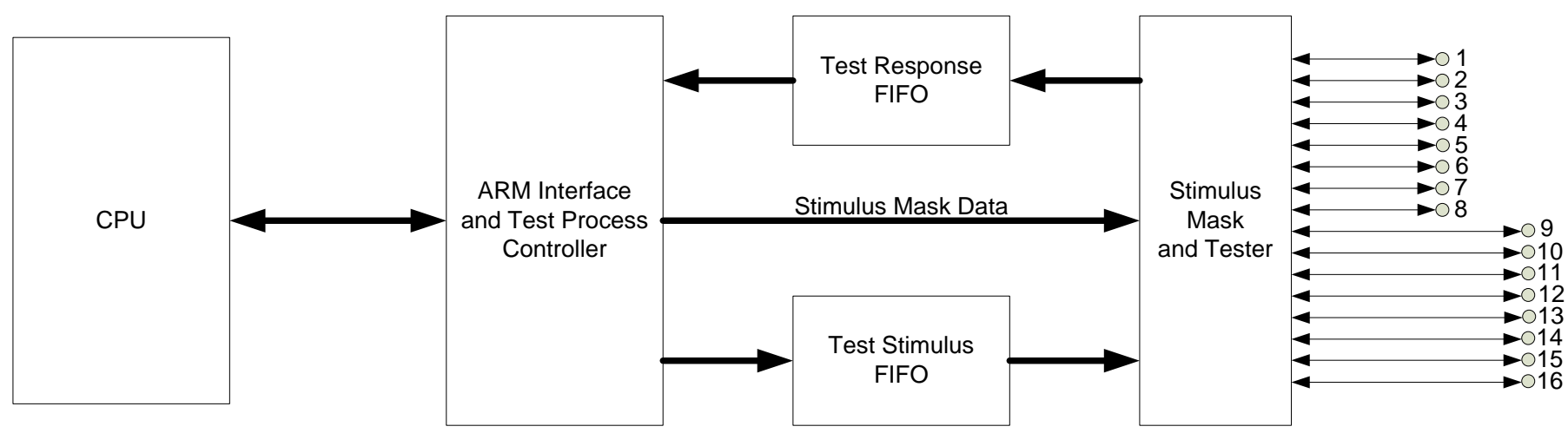

The following diagram is a graphic representation of the process of branch exploration in a target IC with one data input pin (in addition to a clock pin). The responses, which also represent the states, form the raw tree of the FSM, which is processed through branch folding algorithms. This process of exploration and folding is repeated until all branches and states within the limit of the stimulus/response count have been found.

FSM Branch Exploration using a Stimulus/Response Process for a one-bit input

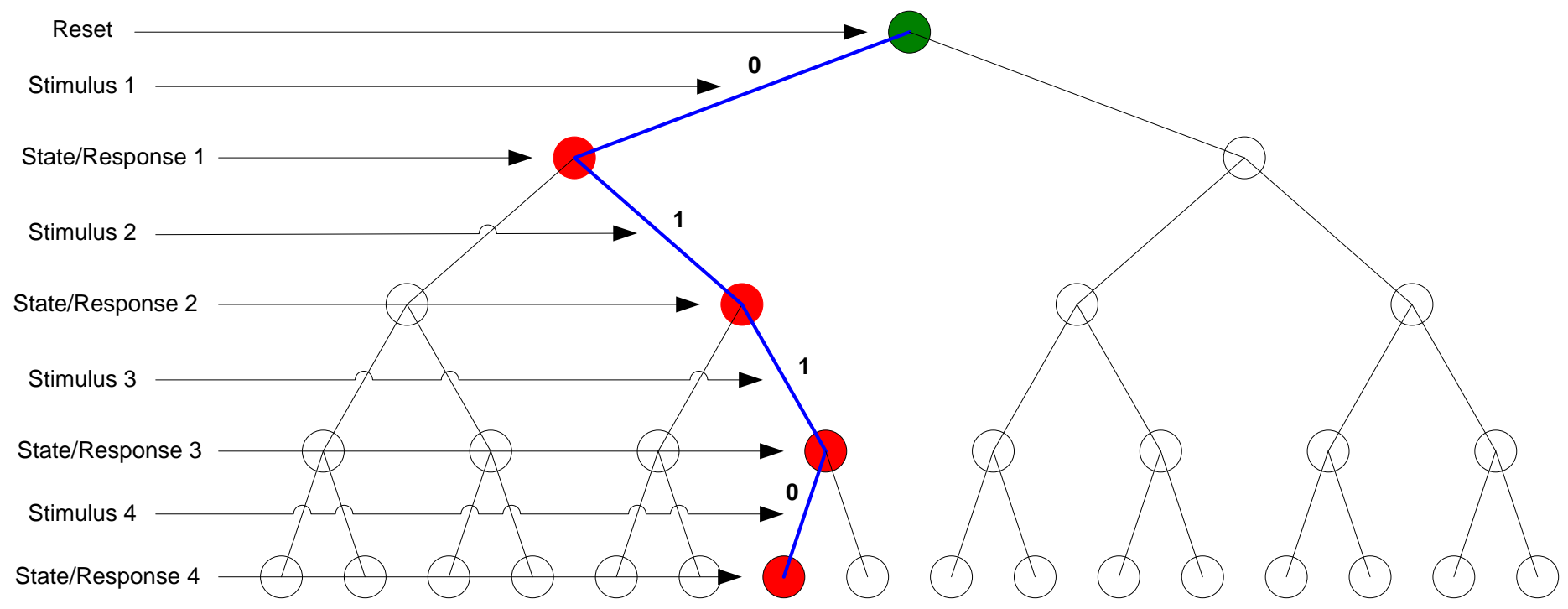




\section{Burst Brute-Force Branch Exploration}

The burst explore approach simply puts the stimulus generation algorithm onto the Testsofa, itself, instead of waiting for the Boss to create the stimulus and send it to the Testsofa through the USB or Ethernet connection. This dramatically reduces the overall time for the exploration process.

The stimulus generator takes a desired node address and creates the input stimulus using an algorithm based on the Address equation in Equation 1 (see appendix B for more details).

$$
\text { Addr }=\sum_{\text {StimIndex }=0}^{\text {StimLength }}\left[2^{\# I n p u t s * \text { StimIndex }}+\left(2^{\# \text { Inputs }}\right)^{(\text {StimLength-StimIndex })}{ }^{\text {Stim }}{ }_{\text {StimIndex }}\right]
$$

Equation 1: Node address, where StimIndex is the position of the stimulus in the stimulus branch and StimLength is the length of the stimulus branch.

The burst explore algorithm is done breadth-wise using a series of spurts. A spurt is a sequence of $2^{\text {\#nput }}$ Reset/Stimulus streams starting from a parent node, one for each possible permutation of the inputs, as shown in Figure 3.

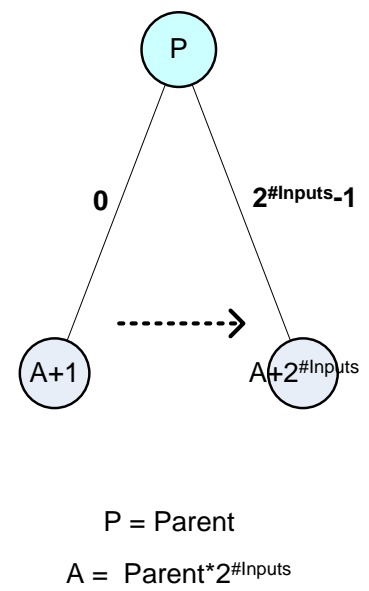

Figure 3: Tree diagram of a spurt.

For non-root starting nodes, the spurt will include the stimulus needed to get from the root to the desired parent node address, which will be inserted between the reset and the spurt stimulus. The following chart, for a 2- input pin IC, depth of 1 branch exploration, gives the tree diagram in the left column, and the testsofa's internally generated stimulus and returned response in the right column.

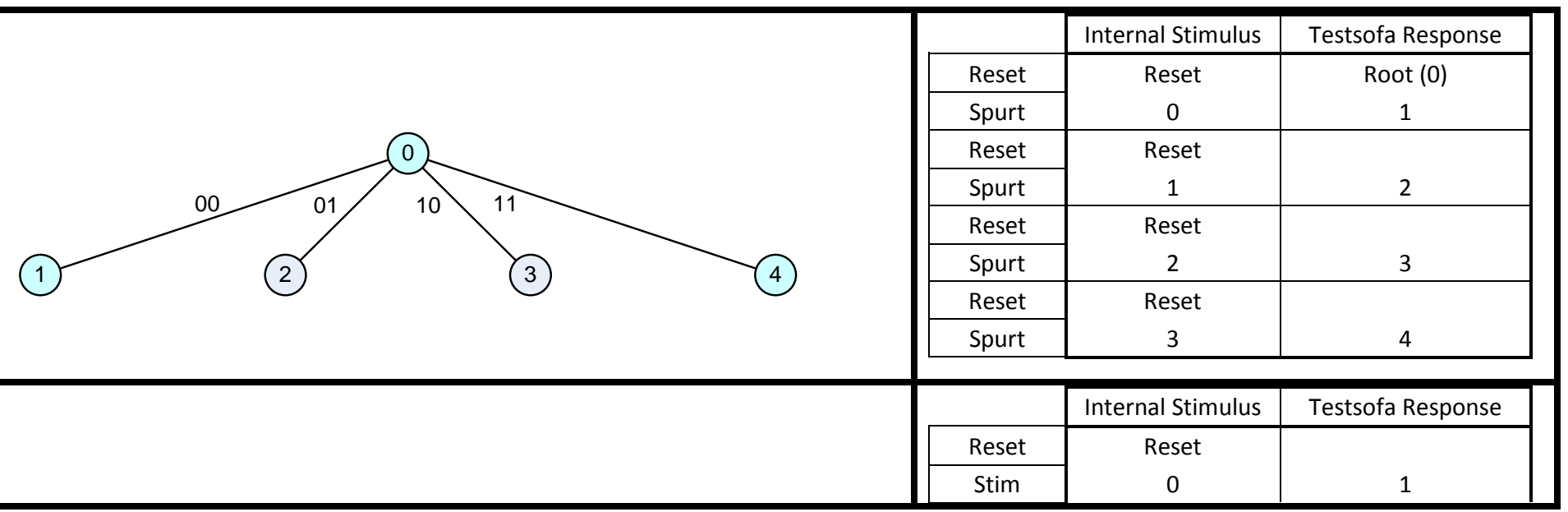




\begin{tabular}{|c|c|c|c|}
\hline & Spurt & 0 & 5 \\
\hline & Reset & Reset & \\
\hline & Stim & 0 & \\
\hline & Spurt & 1 & 6 \\
\hline & Reset & Reset & \\
\hline & Stim & 0 & \\
\hline 111 & Spurt & 2 & 7 \\
\hline & Reset & Reset & \\
\hline & Stim & 0 & \\
\hline & Spurt & 3 & 8 \\
\hline & & Internal Stimulus & Testsofa Response \\
\hline & Reset & Reset & \\
\hline & Stim & 1 & 2 \\
\hline & Spurt & 0 & 9 \\
\hline & Reset & Reset & \\
\hline & Stim & 1 & \\
\hline & Spurt & 1 & 10 \\
\hline & Reset & Reset & \\
\hline & Stim & 1 & \\
\hline (8) (1) (10) (12) & Spurt & 2 & 11 \\
\hline & Reset & Reset & \\
\hline & Stim & 1 & \\
\hline & Spurt & 3 & 12 \\
\hline & & Internal Stimulus & Testsofa Response \\
\hline & Reset & Reset & \\
\hline & Stim & 2 & 3 \\
\hline & Spurt & 0 & 13 \\
\hline & Reset & Reset & \\
\hline & Stim & 2 & \\
\hline & Spurt & 1 & 14 \\
\hline & Reset & Reset & \\
\hline 5 & Stim & 2 & \\
\hline (9) (10) (11) & Spurt & 2 & 15 \\
\hline & Reset & Reset & \\
\hline & Stim & 2 & \\
\hline & Spurt & 3 & 16 \\
\hline & & Internal Stimulus & Testsofa Response \\
\hline & Reset & Reset & \\
\hline & Stim & 3 & 4 \\
\hline & Spurt & 0 & 17 \\
\hline & Reset & Reset & \\
\hline & Stim & 3 & \\
\hline & Spurt & 1 & 18 \\
\hline $00 / 01 / 10$ & Reset & Reset & \\
\hline (5) 6 ( 7 (8) 9 10 11 (12) 13 14 15 & Stim & 3 & \\
\hline (5) (6) (7) (8) (9) (10) (11) (12) (13) (14) (15) (16) & Spurt & 2 & 19 \\
\hline & Reset & Reset & \\
\hline & Stim & 3 & \\
\hline & Spurt & 3 & 20 \\
\hline
\end{tabular}




\section{Communications Format}

The USB communications packet format between the host and the sofa consists of a 16-Byte Header followed by an optional series of 16byte state exploration data sections. Only the Branch Exploration and Burst Exploration command responses contain these data sections.

The USB header has the following format:

USB HEADER

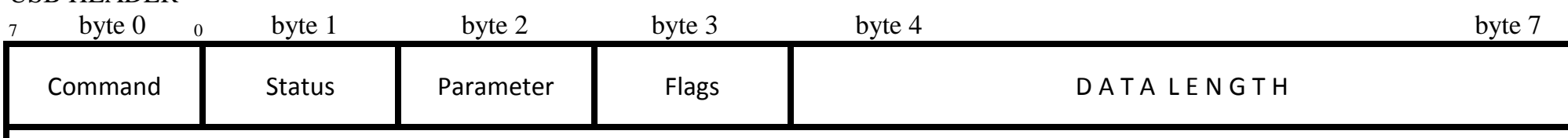

64-bit Start Node Address / FSM Current State / Diagnostic \& Pin Profile Response Data

This 16-byte header is the only structure required to be sent to the sofa from the host (i.e., the sofa will always be expecting to read 16-bytes at a time from the host). Some of the fields of the header are shared between the various host commands, that is, they serve different purposes for different commands. Specifically, the most significant 8 bytes of the header serve as both the Start Node Address and FSM Current State for Burst Exploration commands and contain the response data returned from the sofa for the Pin Profile and Diagnostic Requests. The Parameter byte is also used to specify which state machine the sofa should emulate for the FSM command and to contain the exploration depth for exploration commands (for burst exploration, only depth 1 is supported). The Flags field can be used to indicate Extended Data(by setting the field to 1), meaning that the amount of response data exceeds what can be represented by the 32-bit DataLength field. The additional length (not including what is specified in the DataLength field) is provided by a 4-byte field which will immediately follow the header. The DataLength field is also used by the Pin Profiling Request packet, to inform the sofa as to how many pins the IC being tested has.

In Response packets, the sofa will return the header, containing the same command and parameter values sent to it, the other fields may be modified, depending on the specific command sent.

Along with 0 , the following are valid values for the various fields of the header:

\begin{tabular}{|r|c|c|l|}
\hline \multicolumn{1}{|c|}{ Field } & Value & Length & \multicolumn{1}{c|}{ Comment } \\
\hline Command & 0 & 1 & Reset - instructs the test sofa to perform a reset, there is no response provided to the Host. \\
\hline & 1 & & Pin Profile - requests the \# of input and output pins for the IC unit under test. \\
\hline & 2 & & Branch Exploration - currently unsupported. \\
\hline & 3 & & Burst Exploration - requests a branch exploration of depth 1 from a given node. \\
\hline & 4 & & FSM - requests the Sofa to enter into Finite State Machine emulation mode. \\
\hline & 5 & & Diagnostic - requests information related to the sofa hardware. \\
\hline Status & $0 / 1$ & 1 & Cleared by host, set to 1 by sofa to positively acknowledge requests. \\
\hline & Depth & 1 & Used by Branch/Burst exploration requests to indicate depth to explore to / or to hold error codes. \\
\hline & TableID $\dagger$ & & Used by FSM mode requests to indicate FSM table to use. \\
\hline & Error Code & & If response 'Status’ == 0, contains specific error code, see appendix C. \\
\hline Flags & Bitmap $¥$ & 1 & Indicates FSM mode of operation and / or presence of extended data. \\
\hline High Octet & & 4 & Number of bytes of state exploration data. \\
\hline
\end{tabular}

$\dagger$ Valid Table IDs are:

0: Exit FSM Mode

1: Triangle, 1-pin

2: Tree, 1- pin

3: Tree, 2-pin

4: Cube, 2-pin
$¥$ Valid Bit Settings:

ar $s r-a q s q-x d$

$\begin{array}{llllllll}0 & 0 & 0 & 0 & 0 & 0 & 0 & 0\end{array}$ $a r$ : address, response $s r$ : state, response

$a q$ : address, request $s q$ : state, request

$x d$ : extended data present (see above)

$a r$ and $s r$ set by sofa in burst explore response packets to indicate it is operating in FSM mode and that the first octet of the state exploration data sections contains a node address (if $a r$ is set) or contains 0 , indicating FSM 'state' mode (if $s r$ is set).

$a q$ and $s q$ are set by the host in both FSM Mode and burst explore request packets. In FSM Mode request packets, $a q$ is set to indicate that the host is requesting the sofa to operate in FSM 'Address' mode (meaning that subsequent Burst Exploration requests will provide a 64-bit Start Node Address). When FSM Mode request packets have the $s q$ bit set, this indicates that the host wishes the sofa operate in FSM 'State' mode (meaning that subsequent Burst Exploration requests will provide a 32-bit State value that the sofa should use to index into its State Table). Burst Exploration requests/responses will continue to maintain setting either the $a q$ or $s q$ / ar or sr bit accordingly until the host requests the sofa exit FSM emulation mode by issuing a FSM request with Table ID $=0$. NOTE: THE ar,sr/aq,sq ARE ONLY TO BE SET WHEN OPERATING IN ONE OF THE 2 FSM MODES (Address or State mode), NOT DURING OTHER TIMES. 
Reset

Request Format (Header.Cmd $=0$ )

\begin{tabular}{|c|c|c|c|c|c|}
\hline \multicolumn{1}{c}{ Cmd } & Status & Param & Flags & Datalength LSB & Datalength MSB \\
\hline 00 & 00 & 00 & 00 & & 00_00_00_00 \\
\hline
\end{tabular}

00_00_00_00_00_00_00_00

LSB

The Reset request consists of 16 bytes of Zeroes and instructs the test sofa to perform a reset.

There is no response from the sofa to this command. Following a Reset, the test sofa should come up in non-fsm mode, with no pin profile data and nothing in its usb buffer.

\section{Pin Profile}

Request Format (Header.Cmd $=1$ )

\begin{tabular}{|c|c|c|c|c|}
\multicolumn{1}{c|}{ Cmd } & Status & Param & Dlags & Datalength LSB \\
\hline 01 & 00 & 00 & 00 & Total \# of pins on IC \\
\hline & & 00_00_00_00_00_00_00_00 \\
\hline
\end{tabular}

Response Format

\begin{tabular}{|l|l|l|l|l|}
\hline \multicolumn{1}{|c|}{ Cmd } & Status & Param & Flags & Datalength LSB \\
\hline 01 & $01 / 00$ & $00 / \mathrm{EC}$ & 00 & Total \# of pins on IC \\
\hline \multicolumn{3}{|c|}{ \# of input pins output pins } \\
\hline
\end{tabular}

LSB

If there is no pin physical preset in the test sofa, the sofa will return error code value 5.

\section{Branch Exploration}

Request Format (Header.Cmd $=2$ )

$* *$

** THE BRANCH EXPLORATION COMMAND IS CURRENTLY UNSUPPORTED **

** THE SUPPORTED BURST EXPLORATION COMMAND IS A SUBSET OF THIS**

** COMMAND, BUT WITH A FIXED EXPLORATION DEPTH OF JUST 1 LEVEL **

*** (i.e., will always use a depth level of 1) 
Burst Exploration

Request Format (Header.Cmd = 3, Header. Parameter $=$ depth (1))

Cmd

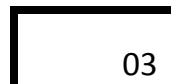

Status

00
Param

01
Flags

$00 / a q, s q$
Datalength LSB

Datalength MSB

00_00_00_00

Start Node Address / Start FSM State

LSB

If the Header.Flags field in the Burst Exploration request packet has either the $a q$ or $s q$ bit set (see above), the host is operating under the assumption that the sofa is in FSM mode (see FSM Command), in which case the bytes beginning at byte 8 in the request header will be used to supply the sofa with either a machine state value to be used by the sofa to lookup a state transition in the appropriate state table (case of $s q$ bit set), or those bytes will contain a start node address (case of $a q$ bit set). If sofa is not currently in FSM mode it should return an error code status (see Appendix C).

NOTE: THE $a q / s q$ BITS ARE ONLY TO BE SET WHEN BY THE HOST WHEN OPERATING IN ONE OF THE 2 FSM MODES (Address or State mode), NOT DURING OTHER TIMES. THE HOST SHOULD CLEAR THE $a r / s r$ bits IN EACH REQUEST.

If a pin profile request has not yet been issued to the test sofa since the last Reset was performed, the test sofa will return an error code value of 4 . 
Burst Exploration

Response Format (Header.Cmd $=3$ Header. Parameter $=$ depth (1))

\begin{tabular}{|c|c|c|c|c|c|}
\hline \multicolumn{1}{c|}{ Cmd } & Status & Param & \multicolumn{1}{l}{ Flags } & Datalength LSB & Datalength MSB \\
\hline 03 & $01 / 00$ & $01 / \mathrm{EC}$ & $00 / a r, s r$ & & xx_xx_xx_xx \\
\hline
\end{tabular}

Start Node Address / Start FSM State

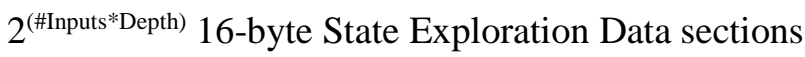

In both normal and FSM modes, the sofa will return a response as indicated above - including the appropriate number of

State Exploration Data sections as represented by the following 16-byte format:

$\begin{array}{lllll}\text { byte } 0 & \text { byte } 1 & \text { byte } 2 & \text { byte } 3 & \text { byte } 4\end{array}$

byte 7

Child Node Address / Child Node FSM State

Input stimulus

Output state

LSB

Under normal operation (i.e., not operating in FSM mode) using the burst exploration request data, the testsofa will

apply the NodeAddress formula (solving for input stimulus, see equation 1 above) to the provided Start Node Address to infer the input stimuli required, from RESET, to bring the sofa to the state represented by the address. The sofa will then generate stimuli for all possible input combinations to effectively generate child nodes in a state tree. For each child node, its corresponding input stimulus (including RESET).will be applied, the sofa output state will be recorded and the node address of the child will be generated (via the NodeAddress formula). Note that before applying each child node's input stimulus, a RESET must be done and the full stream of input stimuli represented by the provided parent node address must be applied.

For normal burst explorations as well as for FSM Address mode, the sofa will return a 64-bit Child Node Address. For FSM State mode responses, it will return the Child Node State (same value as OutputState).

If the Header.Flags field in the Burst Exploration request packet had either the $a q$ or $s q$ bit set the host is operating under the assumption that the sofa is in FSM mode (see FSM Command), in which case the bytes beginning at byte 8 in the request header will be used to supply the sofa with either a machine state value to be used by the sofa to lookup a state transition in the appropriate state table (case of $s q$ bit set), or those bytes will contain a start node address (case of $a q$ bit set). If sofa is not currently in FSM mode it should return a 0 in the Status field to indicate it could not complete the request and an error code status (see Appendix C), otherwise the sofa should return the appropriate fsm mode bit (ar or $s r$ ) in its responses.

In FSM State mode, the sofa will not have to infer any input stimuli to bring the sofa to a particular state, and will not need to do a RESET, it will simply use the provided FSM Current State to lookup the state transitions for all possible input stimuli for the particular Finite State Machine it is emulating. In FSM Address mode, the sofa will operate as if under normal operation (see above) except that it will not need to apply physical RESETs or INPUT transitions to the hardware, but will instead apply these logically to the currently active FSM table.

The total number of state information data sections returned is dependent upon the number of input pins, in accordance

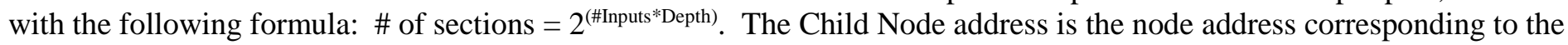
complete set of input stimuli from RESET and including the associated input stimulus. The input stimulus field contains the input stimuli used by the sofa to generated the associated output state (i.e., for 3 inputs, this field can contain a value from $000 \mathrm{~b}$ to $111 \mathrm{~b}(7)$ ). The output state field is the state of the IC outputs after the input stimulus is applied (following a RESET).

\section{NOTE: THE ar/sr BITS ARE ONLY TO BE SET BY THE SOFA WHEN OPERATING IN ONE OF THE 2 FSM MODES (AddresS or State} mode), NOT DURING OTHER TIMES. THE SOFA SHOULD CLEAR THE aq/sq bits IN EACH RESPONSE.

If a pin profile request has not yet been issued to the test sofa since the last Reset was performed, the test sofa will return an error code value of 4 . 
FSM Command

Request Format (Header.Cmd = 4, Header. Param = tableID)

\begin{tabular}{|c|c|c|c|cc|}
\hline \multicolumn{1}{c|}{ Cmd } & Status & Param & Flags & Datalength LSB & Datalength MSB \\
\hline 04 & 00 & Table ID & 0x08 / 0x04 & 00_00_00_00 \\
\hline & & 00_00_00_00_00_00_00_00 & \\
\hline
\end{tabular}

LSB

The FSM command is used to request the sofa to emulate a finite state machine, using the table ID to do so. In this mode, the sofa will respond to burst exploration commands by retrieving output states from a state machine table. The Flags field indicates whether to operate in 'Address' or 'State' mode (see $a q / s q$ bits above).

The following table IDs are currently supported:
0: Exit FSM Mode
1: Triangle, 1-pin
2: Tree, 1- pin
3: Tree, 2-pin
4: Cube, 2-pin

Response Format

\begin{tabular}{|c|c|c|c|cc|}
\multicolumn{1}{c|}{ Cmd } & Status & Param & Flags & Datalength LSB & Datalength MSB \\
\hline 04 & $01 / 00$ & Table ID / EC & 0x80 / 0x40 & 00_00_00_00 \\
\hline
\end{tabular}

LSB

If the sofa supports this command and table ID, the Header.Status field will be returned as 1 , otherwise it will be returned as 0 and an appropriate error code in the 'Param' field (see Appendix C). The sofa will acknowledge being put into either 'Address' or 'State' mode via appropriate setting of the Flags field (see $a r / s r$ bits above). If a pin profile request has not yet been issued to the test sofa since the last Reset was performed, the test sofa will return an error code value of 4 .

\section{Diagnostics}

Request Format $($ Header.Cmd $=5)$

\begin{tabular}{|c|c|c|c|c|c|}
\hline $\mathrm{Cmd}$ & Status & Param & Flags & Datalength LSB & Datalength MSB \\
\hline 05 & 00 & 00 & 00 & & \\
\hline
\end{tabular}

LSB

The Diagnostic command is used to request information related to the sofa hardware:

Response Format

\begin{tabular}{|c|c|c|c|c|c|}
\hline \multicolumn{1}{|c|}{ Cmd } & Status & Param & Flags & Datalength LSB & Datalength MSB \\
\hline 05 & $01 / 00$ & $00 / E C$ & 00 & $00 \_00 \_00 \_00$ \\
\hline \multicolumn{2}{|c|}{ Sofa Firmware Version } & \multicolumn{2}{|c|}{ Sofa State } & unused & unused \\
\hline
\end{tabular}


The following example shows the byte stream for several commands:

Setting FSM 'State' Mode with Table 3

\begin{tabular}{rllllllll}
\hline$--->$ & $0 \times 04$ & $0 \times 00$ & $0 \times 03$ & $0 \times 04$ & $0 \times 00$ & $0 \times 00$ & $0 \times 00$ & $0 \times 00$ \\
$0 \times 00$ & $0 \times 00$ & $0 \times 00$ & $0 \times 00$ & $0 \times 00$ & $0 \times 00$ & $0 \times 00$ & $0 \times 00$ \\
& & & & & & & & \\
& $0 \times 04$ & $0 \times 01$ & $0 \times 03$ & $0 \times 40$ & $0 \times 00$ & $0 \times 00$ & $0 \times 00$ & $0 \times 00$ \\
$0 \times 00$ & $0 \times 00$ & $0 \times 00$ & $0 \times 00$ & $0 \times 00$ & $0 \times 00$ & $0 \times 00$ & $0 \times 00$
\end{tabular}

successfully set sofa fsm mode to use '2-pin tree' state machine in 'State' mode.

Requesting Pin Profile (for a 16-pin IC):

$\begin{array}{lllllllll}---> & 0 \times 01 & 0 \times 00 & 0 \times 00 & 0 \times 00 & 0 \times 10 & 0 \times 00 & 0 \times 00 & 0 \times 00\end{array}$

$\begin{array}{lllllllll}0 \times 00 & 0 \times 00 & 0 \times 00 & 0 \times 00 & 0 \times 00 & 0 \times 00 & 0 \times 00 & 0 \times 00\end{array}$

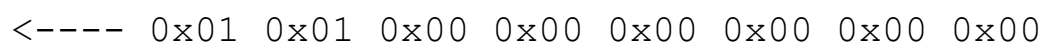

$0 \times 02 \quad 0 \times 00 \quad 0 \times 00 \quad 0 \times 00 \quad 0 \times 03 \quad 0 \times 00 \quad 0 \times 00 \quad 0 \times 00$

The response indicates that there are 2 input and 3 output pins.

Requesting Burst Exploration (non-FSM mode) from node $0 \times 56783322$ to depth of 1

---> $0 \times 03$ 0x00 0x01 $0 \times 00 \quad 0 \times 00$ 0x00 $0 \times 00 \quad 0 \times 00$

$0 \times 22 \quad 0 \times 33 \quad 0 \times 78 \quad 0 \times 56 \quad 0 \times 00 \quad 0 \times 00 \quad 0 \times 00 \quad 0 \times 00$

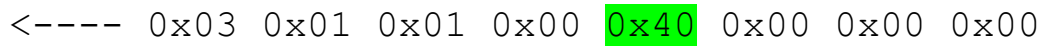

$\begin{array}{lllllllll}0 \times 22 & 0 \times 33 & 0 \times 78 & 0 \times 56 & 0 \times 00 & 0 \times 00 & 0 \times 00 & 0 \times 00\end{array}$

sofa successfully responded with data length indication of $0 \times 40$ bytes of state information

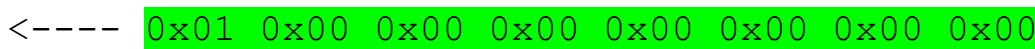

$\begin{array}{lllllllll}0 \times 00 & 0 \times 00 & 0 \times 00 & 0 \times 00 & 0 \times 01 & 0 \times 00 & 0 \times 00 & 0 \times 00\end{array}$

$\begin{array}{lllllllll}0 \times 02 & 0 \times 00 & 0 \times 00 & 0 \times 00 & 0 \times 00 & 0 \times 00 & 0 \times 00 & 0 \times 00\end{array}$

$\begin{array}{llllllll}0 \times 01 & 0 \times 00 & 0 \times 00 & 0 \times 00 & 0 x 02 & 0 \times 00 & 0 x 00 & 0 x 00\end{array}$

$\begin{array}{lllllllll}0 \times 03 & 0 \times 00 & 0 \times 00 & 0 \times 00 & 0 \times 00 & 0 \times 00 & 0 \times 00 & 0 \times 00\end{array}$

$\begin{array}{lllllllll}0 \times 02 & 0 \times 00 & 0 \times 00 & 0 x 00 & 0 x 03 & 0 x 00 & 0 x 00 & 0 x 00\end{array}$

$\begin{array}{lllllllll}0 \times 04 & 0 \times 00 & 0 \times 00 & 0 \times 00 & 0 \times 00 & 0 \times 00 & 0 \times 00 & 0 \times 00\end{array}$

$0 \times 03 \quad 0 \times 00 \quad 0 \times 00 \quad 0 \times 00 \quad 0 \times 04 \quad 0 \times 00 \quad 0 \times 00 \quad 0 \times 00$

host read of $0 \times 40$ bytes of response of state information

The response indicates:

node 1 produces state 1 with a stimulus of 0 (both inputs low).

node 2 produces state 2 with a stimulus of 1 (input 1 high, input 2 low).

node 3 produces state 3 with a stimulus of 2 (input 1 low, input 2 high).

node 4 produces state 4 with a stimulus of 3 (input 1 high, input 2 high). 


\section{Appendix A:}

\section{Example for 74HC194 Bidirectional Universal Shift Register chip:}

\section{Pin Profiling:}

Request Types:

Empty: UUT pin-profile is unknown. Pin-profiling will need to done, which can be time-consuming.

Filled: UUT pin-profile is known, so pin-profiling process can be skipped.

Request (Empty)

\begin{tabular}{|c|c|c|c|c|c|c|c|c|c|}
\hline $\mathbf{0}$ & $\mathbf{1 6}$ & $\mathbf{0 0}$ & $\mathbf{0 0}$ & $\mathbf{0 0}$ & $\mathbf{0 0}$ & $\mathbf{0 0}$ & $\mathbf{0 0}$ & $\mathbf{0 0}$ & $\mathbf{0 0}$ \\
\hline
\end{tabular}

Response
\begin{tabular}{|l|c|c|}
\hline $\mathbf{0}$ & $\mathbf{8}$ & $\mathbf{4}$ \\
\hline
\end{tabular}

\section{Branch Exploration through Stimulus and Response:}

Stimulus:

\begin{tabular}{|c|c|c|c|c|c|c|c|c|c|c|c|c|}
\hline 1 & 16 & 10 & 65 & 64 & 64 & 64 & 128 & 128 & 128 & 64 & 64 & 64 \\
\hline
\end{tabular}

\begin{tabular}{|c|c|c|c|c|c|c|c|c|c|c|c|c|c|}
\hline \multirow{2}{*}{ Stimulus } & \multicolumn{8}{|c|}{ UUT Inputs } & \multicolumn{4}{|c|}{ UUT Outputs } & \multirow{2}{*}{ Response } \\
\hline & 8 & 7 & 6 & 5 & 4 & 3 & 2 & 1 & 4 & 3 & 2 & 1 & \\
\hline Rst & 0 & 0 & 0 & 0 & 0 & 0 & 0 & 0 & 0 & 0 & 0 & 0 & 0 \\
\hline 65 & 0 & 1 & 0 & 0 & 0 & 0 & 0 & 1 & 0 & 0 & 0 & 0 & 0 \\
\hline 64 & 0 & 1 & 0 & 0 & 0 & 0 & 0 & 0 & 1 & 0 & 0 & 0 & 8 \\
\hline 64 & 0 & 1 & 0 & 0 & 0 & 0 & 0 & 0 & 0 & 1 & 0 & 0 & 4 \\
\hline 64 & 0 & 1 & 0 & 0 & 0 & 0 & 0 & 0 & 0 & 0 & 1 & 0 & 2 \\
\hline 128 & 1 & 0 & 0 & 0 & 0 & 0 & 0 & 0 & 0 & 0 & 0 & 1 & 1 \\
\hline 128 & 1 & 0 & 0 & 0 & 0 & 0 & 0 & 0 & 0 & 0 & 1 & 0 & 2 \\
\hline 128 & 1 & 0 & 0 & 0 & 0 & 0 & 0 & 0 & 0 & 1 & 0 & 0 & 4 \\
\hline 64 & 0 & 1 & 0 & 0 & 0 & 0 & 0 & 0 & 1 & 0 & 0 & 0 & 8 \\
\hline 64 & 0 & 1 & 0 & 0 & 0 & 0 & 0 & 0 & 0 & 1 & 0 & 0 & 4 \\
\hline 64 & 0 & 1 & 0 & 0 & 0 & 0 & 0 & 0 & 0 & 0 & 1 & 0 & 2 \\
\hline
\end{tabular}




\section{Appendix B:}

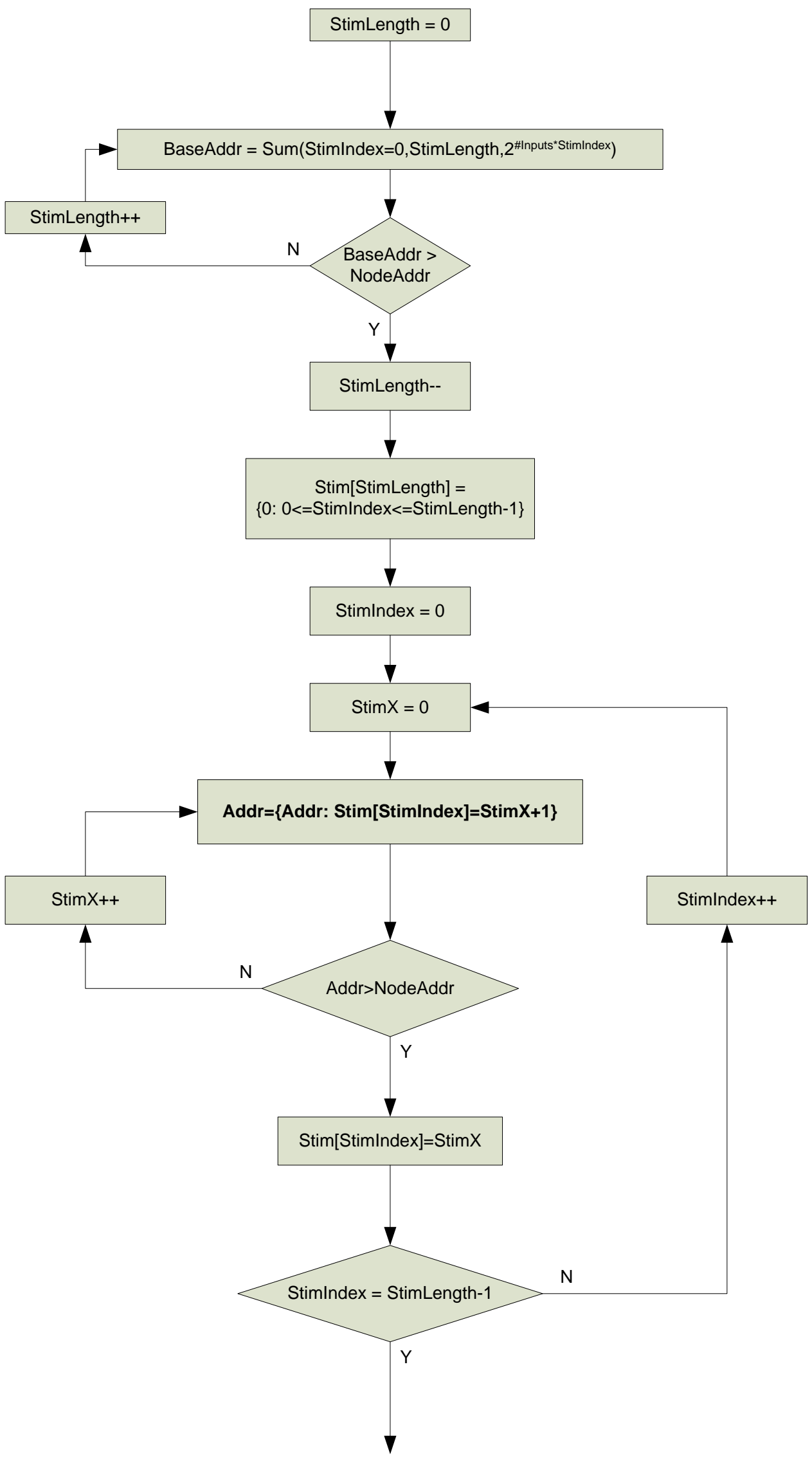




\section{Appendix C- Error Codes}

If the test sofa cannot execute a host request, it will return a value of 0 in the response packet 'Status' field and one of the following values in the 'Parameter' field:

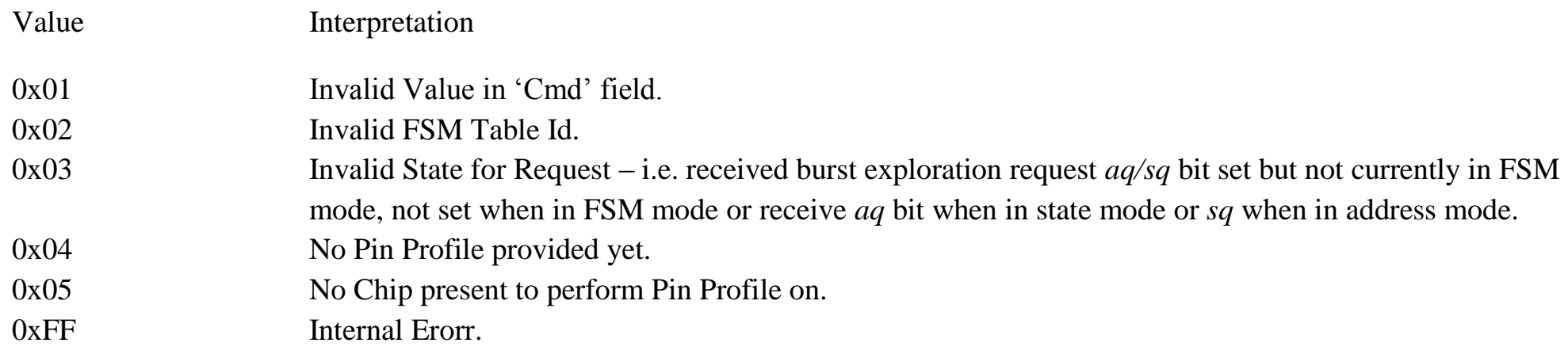

